\title{
Microfluidics in coagulation monitoring devices: A mini review
}

Leanne Harris

Technological University Dublin, leanne.harris@tudublin.ie

Anthony J. Killard

University of the West of England, Bristol, tony.klllard@uwe.ac.uk

Follow this and additional works at: https://arrow.tudublin.ie/scschbioart

Part of the Biology Commons

\section{Recommended Citation}

Harris, Leanne \& Killard, Anthony. (2018). Microfluidics in coagulation monitoring devices: A mini review. Analytical Methods. 10. 10.1039/C8AY01230J.

This Article is brought to you for free and open access by the School of Biological Sciences at ARROW@TU Dublin. It has been accepted for inclusion in Articles by an authorized administrator of ARROW@TU Dublin. For more information, please contact arrow.admin@tudublin.ie, aisling.coyne@tudublin.ie,gerard.connolly@tudublin.ie.

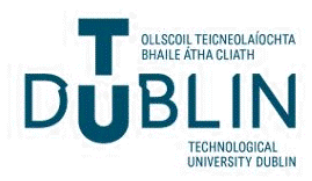




\title{
Microfluidics in coagulation monitoring devices: a mini review
}

Cite this: Anal. Methods, 2018, 10, 3714

\author{
Leanne F. Harris $\mathbb{D D}^{* a}$ and Anthony J. Killard (iD ${ }^{\mathrm{b}}$
}

Received 30th May 2018

Accepted 6th July 2018

DOI: 10.1039/c8ay01230j

rsc.li/methods

\begin{abstract}
Diagnosis and monitoring of disorders of coagulation and disturbances in haemostasis has been around for many years. Thrombotic and bleeding disorders are amongst the leading causes of mortality in the developed world, thus driving research in this area. The simplicity with which miniaturised devices operate and their application to the study of coagulation and haemostasis offer potential advantages over traditional testing, such as improvements in clinical decision making and ultimately patient care. This review looks at progress in microfluidic device development in the field of haemostasis and coagulation biology.
\end{abstract}

\section{Introduction}

Microfluidic devices allow for the identification of a range of diagnostic targets inclusive of metabolites, proteins, nucleic acids, cells and pathogens. ${ }^{1}$ The application of miniaturised point of care (POC) technologies is well established in coagulation and haemostasis. Commercial devices in this space have focused on monitoring of anticoagulant therapies to target bleeding and thrombotic disorders and have successfully contributed to the point-of-care testing (POCT) market, which was estimated to be worth approximately US $\$ 5.6$ billion in $2011 .^{2}$ In contrast, microfluidic research in this area is focused on the measurement of platelet activity and the identification of coagulation proteins to aid in the study of blood function to inform clinical decisions around haemostatic disorders. ${ }^{1,3}$

Microfluidics involves the handling of small volumes of liquid within channels that are mere micrometres $(10-100 \mu \mathrm{m})$ in dimension. ${ }^{4}$ The major advantages of these miniaturised devices include low cost due to the use of disposable materials, rapid turnaround times, and enhanced sensitivity and specificity. ${ }^{1,4}$ The low-cost aspect of these technologies is in part related to the type of material used for their fabrication, which can range from polymer or plastic substrates to paper., ${ }^{5,6}$ There is also a transition away from large scale clean room fabrication methodologies, in preference for low-cost equipment such as laser cutters and 3-D printers, that are highly compatible for the manufacture of paper or plastic chips. This presents the life science community with opportunities to exploit lab on a chip (LOC) technologies for high throughput analysis of biological samples, that were previously limited or even inaccessible. ${ }^{5}$

${ }^{a}$ Dublin Institute of Technology, School of Biological Sciences, Kevin Street, Dublin 8, Ireland. E-mail: leanne.harris@dit.ie; Tel: +353 14024636

${ }^{b}$ Department of Applied Sciences, Faculty of Health and Applied Sciences, University of the West of England, Frenchay Campus, Coldharbour Lane, Bristol BS16 1QY, UK. E-mail: tony.klllard@uwe.ac.uk; Tel: +44 (0)1173282147
The biology of blood coagulation is not without complexity involving a range of clotting proteins, each exerting their effect in the next step of the clotting cascade. ${ }^{7}$ Divided into two key pathways, the intrinsic pathway is activated by damaged vessels that expose negatively charged activators causing the sequential activation of clotting factor proteins. It is this activation of clotting factors that ultimately results in the formation of a fibrin clot. The alternative extrinsic pathway results from damage to the endothelium releasing tissue factor, the trigger for platelet activation and fibrin polymerisation. ${ }^{7,8}$

It is these pathways that are targeted by the traditional clotbased assays such as the prothrombin time (PT), an assay that measures the length of time it takes for clot formation through the extrinsic pathway. The activated partial thromboplastin time (aPTT) and activated clotting time (ACT) tests replicate clotting via the intrinsic pathway. While the thrombin clotting time (TCT) involves the addition of excess thrombin to a sample in vitro to stimulate clot formation. ${ }^{9}$

POC technology offers the opportunity to evaluate and monitor the complexity of the coagulation pathways. As outlined by Lancé the ideal coagulation test should be a reliable, rapid and easy to perform test that gives an accurate indication of the risk of bleeding or clotting. ${ }^{10}$ Researchers can monitor blood in vitro, blood flow under normal conditions and blood flow in an activated sample where coagulation has been imitated, thus mimicking thrombotic events. Innovation in channel design and geometry and manipulation of channel dimensions have allowed for enhanced insight into the complex process of blood coagulation., ${ }^{3,11}$ The majority of monitoring devices on the market do not account for the dynamics of blood flow, which is a significant contributor to platelet function and thrombosis. ${ }^{12}$ However a number of devices in the literature report their incorporation of shear force into their devices to mimic the blood flow of arteries and vessels. They achieve this 
imitation of in vivo vessel dynamics through the use of flow chambers with syringe pumps or plate rollers. ${ }^{11-15}$

In this review we present a snapshot of recent and current microfluidic technologies reported in the literature, that focus on the incorporation of assays specific for the study of haemostasis and coagulation. The devices reported include assays that concentrate, not only on newer methods for monitoring inhibitors of clotting, but microfluidics that can demonstrate the physical process of clot formation, tests that yield information on the mechanisms of platelet function, and clotting factor assays that unearth how individual proteins are impacted in haemostatic disorders.

\section{Microfluidics for anticoagulant monitoring}

The demand for POC devices in coagulation testing was prompted by the need for anticoagulant monitoring after surgery. ${ }^{\mathbf{1 6}}$ The rapid turnaround times achieved with POC devices compared to conventional benchtop instruments played a significant role in their commercial success, due to the ease with which they could be implemented into emergency room settings and conveniently at the patient's bedside.

Microfluidic devices commonly used in the management of anticoagulant therapy incorporate the traditional assays as aforementioned such as the PT, aPTT, ACT and TCT. ${ }^{17}$ The shortcomings of conventional clot-based assays have been reviewed extensively in the literature, in that they do not accurately reflect the entire clotting process, rather one particular point in the cascade, which is the time to clot formation. ${ }^{\mathbf{1 0}}$ Clotbased assays are also renowned for their inter-laboratory variability and limited ranges of measurement. ${ }^{18-20}$

They did however contribute to our understanding of the clotting process and their simple mode of operation positioned them as the ideal candidates for transition into automated laboratory coagulation analysers and subsequently into miniaturised POC devices.

Commercially available microfluidic assays incorporating these conventional clotting assays include the HemoSense INRatio PT/INR system (HemoSense Inc.) which uses electrochemical impedance to measure clotting between printed electrodes on a disposable polymer strip. ${ }^{21}$ The i-STAT (Abbott) is another electrochemical impedance-based device used to measure PT and ACT. ${ }^{2}$ The Xprecia Stride ${ }^{\circledR}$ Coagulation Analyser (Siemens Healthcare $\mathrm{GmbH}$ ) uses electrochemical technology and amperometry for the detection of thrombin activity and has received plaudits for its high quality and innovative ergonomic design. ${ }^{22}$ The combined effect of mechanical and electrochemical detection is also observed in several microfluidic devices on the market that ultimately measure fibrin formation. ${ }^{9}$

Optical detection is another method used for the measurement of clotting time in response to anticoagulation. For example, prior to the development of the Coaguchek XS (Roche Diagnostics International Ltd.), which is an electrochemical impedance-based device, the CoaguChek S (Roche Diagnostics
International Ltd.) incorporated iron filings into a strip that interacted with an electromagnetic field. The filings oscillated until clot formation whereby movement ceased which was detected optically to return the clotting time. ${ }^{23}$ Other devices that followed suit included Hemochron (ITC), ProTime (ITC) and GEM PCL (Instrumentation Laboratory) to name but a few. ${ }^{9}$

The devices outlined above are targeted towards monitoring anticoagulant therapies such as warfarin (vitamin $\mathrm{K}$ antagonist), unfractionated heparin (UFH) or low molecular weight heparin (LMWH). The assays incorporated into these miniaturised devices trigger the intrinsic or extrinsic pathways which involve many clotting proteins, resulting in variable results. The traditional anticoagulant drugs themselves suffer from drawbacks. Anticoagulation with vitamin $\mathrm{K}$ antagonists such as warfarin, is reported to be beyond the therapeutic range almost $50 \%$ of the time. ${ }^{24}$ While heparins, known for their narrow therapeutic windows and unpredictable dose response profiles, can return shortened or prolonged clot times leading to over- or under-dosing of patients. ${ }^{25}$ As a result, more specific targeted assays are required that do not measure the entire clotting cascade.

A polymer microfluidic device was reported in the literature that monitors UFH and LMWH therapy by targeting factor $\mathrm{Xa}(\mathrm{FXa}) .^{18} \mathrm{FXa}$ is a coagulation protein that is at a critical position in the cascade where the intrinsic and extrinsic pathways meet. ${ }^{26,27}$ The disposable laminated microfluidic chip (Fig. 1) uses fluorescence detection, capillary fill of the sample, a volume of $10 \mu \mathrm{l}$ and a result is returned within 60 seconds. A major advantage of this technology is the measurement of FXa, which bypasses the intrinsic and extrinsic pathways in the measurement of anticoagulant effect, thereby enhancing specificity.

UFH has been on the market for many years, but it presents with unpredictable pharmacokinetics and pharmacodynamics. ${ }^{26}$ As a result pharmaceutical companies are taking advantage of the gap in the market and are driving the development of more specific inhibitors of clotting proteins, which include thrombin or factor IIa (FIIa) and FXa. ${ }^{28}$ While these newer drugs may offer improvements in sensitivity and predictability thus minimising the need for monitoring, it will

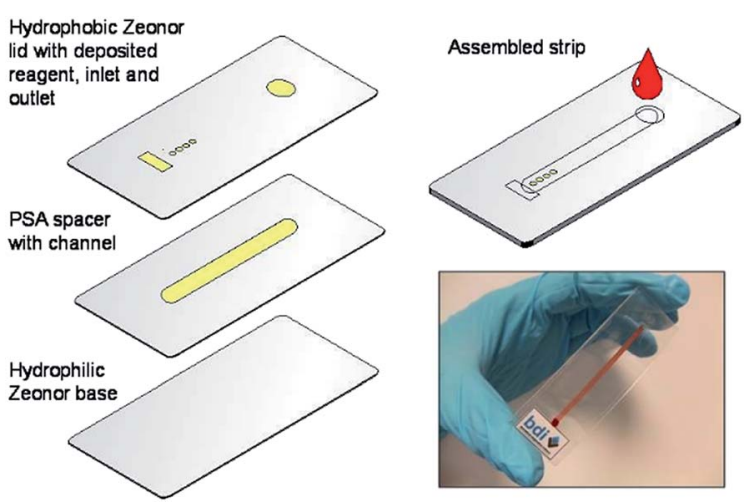

Fig. 1 Schematic and photograph of the microfluidic anti-Xa assay device (reproduced with permission, Harris et al. 2013, RSC). ${ }^{18}$ 
always be required for certain patient cohorts where complications exist. ${ }^{24,25}$

Inhibitors of FXIIa and FXIa have also been recommended for inhibition of thrombosis with a microfluidic device that operates on the principle of contact activation of coagulation. ${ }^{1329}$ An eight-channel device comprised of PDMS (poly(dimethylsiloxane)) was coated with collagen bound to kaolin for activation of the intrinsic pathway. PDMS, an organic polymer is flexible, biocompatible and confers excellent optical properties. $^{2}$ Thrombus structure including platelet and fibrin formation was observed on-chip. Platelet inhibitors (MRS 2197 and 2-MeSAMP) reduced platelet and fibrin deposition, indicating potential for application to studies of FXIIa/FXIa antithrombotics which may offer enhanced specificity over traditional drugs in the future. ${ }^{13}$

\section{Haemostasis 'on a chip'}

Recent research in this area reveals a move away from the conventional clotting time assays for the reasons previously outlined. More specific and detailed analysis of the haemostatic system is required to better understand disorders of coagulation including those disorders resulting from factor deficiencies.

A recent PDMS microchip (Fig. 2) was used to demonstrate the impact of tissue factor (FVII/IIa) on blood clot formation and blood flow. Through manipulation of tissue factor localisation and surface density within the device, platelet deposition, thrombin generation, and fibrin accumulation were analysed. Due to the additional computational modelling carried out, valuable insights were gained into the role of TF in clot formation and resistance to blood flow. ${ }^{30}$

Real-time monitoring of haemostasis using a 12-channel PDMS chip to mimic small blood vessels was also presented by Jain et al. who additionally indicated that shear gradient as opposed to shear rate was responsible for activation of the clotting process. ${ }^{12}$ With the incorporation of mathematical modelling, the antithrombotic effects were observed in realtime, ideal for application to hospital and clinical settings.

Microfluidic devices for cell selection and sorting, in particular for whole blood samples where there is often the requirement to separate red blood cells (RBCs) from plasma (often by centrifugal sedimentation) have been reported extensively in the literature. ${ }^{1,31-33}$ In line with this, blood screening

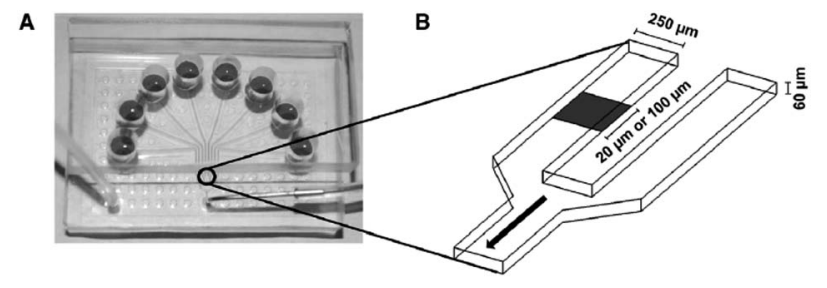

Fig. 2 Photographic (A) and 2-D schematic (B) representation of PDMS microfluidic device used in the determination of blood clot structure and resistance under venous shear (reproduced with permission, Govindarajan et al. 2017, Cell Press). ${ }^{30}$
A
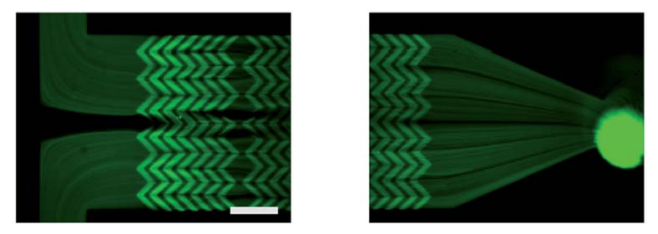

B
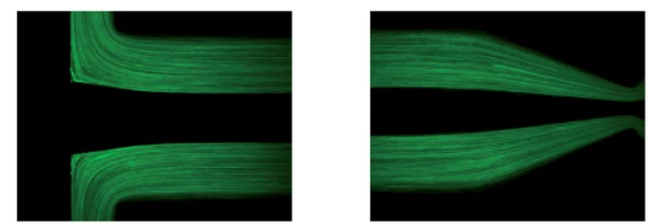

Fig. 3 Suspension of PBS (phosphate buffered saline) with fluorescent beads to illustrate the structure of the microfluidic herringbone mixer with (A) and without (B) herringbone features (reproduced with permission, Lehmann et al. 2018, AIP Publishing). ${ }^{63}$

has also been adapted into a simple paper-based POC assay that incorporates sample separation. The porous cellulose membrane separates plasma from the red blood cells and the colour of the RBCs is used to indicate whether the patient falls within the recommended range for their condition. ${ }^{34}$

Within haemostasis research there is a drive to develop assays that focus on the global measurement of coagulation. Viscoelastic tests such as thromboelastography (TEG) and thromboelastometry (ROTEM) are techniques that result in tracings that reflect the development of a blood clot over time. ${ }^{\mathbf{1 0}}$ Using oscillating sheer force these techniques generate more specific and detailed information on both the clotting process and fibrinolysis, than the traditional clot-based assays. ${ }^{9,35}$ The TEG ${ }^{\circledR}$ Platelet Mapping ${ }^{\mathrm{TM}}$ assay (Haemonetics ${ }^{\circledR}$ Corporation) uses small volumes of blood, while measuring both clot strength and platelet function in patients who are at cardiovascular risk. ${ }^{36}$ For the treatment of postoperative bleeding after cardiac surgery, TEG and ROTEM have been recommended due to their minimal blood requirement, assay versatility and their contribution to rapid clinical decision making. ${ }^{37}$

ClotChip is a microfluidic device reported recently in the literature as being capable of capturing the dynamics of the clotting process using dielectric spectroscopy. ${ }^{38}$ Interestingly, the authors compared their results to ROTEM producing a strong positive correlation, indicating significant potential for monitoring the overall clotting process, rendering it a global assay of haemostasis.

Quartz crystal microbalances (QCM) have featured significantly within the group of technologies that can provide global measurements of haemostasis. They are resonator-based methods that can detect physical changes at its surface. Due to this property and its ultrasensitivity, QCM has been used historically to measure of the mass of films or substances attached to its surface. ${ }^{39}$ It has an additional feature, in that it can measure the viscoelasticity of the target substance at its surface. $^{40}$

QCM has been used in conjunction with standard clot-based assays (PT, aPTT and TCT). ${ }^{\mathbf{3 9 4 - 4 3}}$ While the tests used act as activators of clotting, it is the QCM interface that allows for detailed analysis of the mechanics and kinetics of clot 
formation. Additional information on fibrin fibre diameter and fibre density, which correlates to the rate of clot formation, can also be elucidated using QCM, yet another benefit of this increasingly popular next-generation technology. ${ }^{44}$

Sinn et al. employed QCM methodologies to establish a platelet aggregation assay with significant differences in platelet aggregation observed in both uncoated and fibrinogen coated sensors. ${ }^{45}$ With further development QCM could prove a useful alternative to platelet aggregometry.

Applications of QCM with dissipation (QCM-D) to anticoagulant monitoring has been highlighted in the literature. ${ }^{46} \mathrm{QCM}$ $\mathrm{D}$ and aPTT analysis of samples from patients receiving dabigatran was carried out, returning good correlations $\left(R^{2}=0.99\right)$ between the two methods. QCM-D offers an ultrasensitive method for monitoring the patient response to oral anticoagulant therapy.

\section{Factor specific assays}

Assays that allow for targeting specific coagulation factors afford the opportunity to study the specific roles of clotting proteins in the haemostatic process. Additionally, they yield insight into the behaviours of clotting proteins in diseased states. Specific targets for POC development are not exclusive to clotting factors such as thrombin and fibrinogen but also include fibrinolytic products such as D-dimer or fibrinogen degradation products (FDPs), important in diagnosing thrombotic events or disseminated intravascular coagulation (DIC). ${ }^{9}$

Thrombin is a popular target in coagulation research. A nanochannel device using a nanoporous membrane on chip was reported as successfully measuring thrombin within clinically relevant picomolar ranges. ${ }^{47}$ The device incorporated aptamer/thrombin recognition, which presents with significant advantages due to the high affinity and stability associated with these synthetic nucleic acid sequences. Voltammetric measurement was then employed for the diagnosis of coagulation abnormalities.

Other label-free biosensors for thrombin detection that incorporate aptamers, use poly(pyrrole-nitrilotriacetic acid) ${ }^{48}$ films and indium tin oxide substrates ${ }^{49}$ which show excellent thrombin binding. These high-performance sensors, or aptasensors, provide opportunities to detect thrombin in whole blood with a knock-on effect for disease diagnosis.

Immunoassays are typically employed to detect D-dimer, protein $\mathrm{S}$ and antithrombin. ${ }^{50} \mathrm{D}$-dimer and FDPs are indicative of clot dissolution and the breakdown of fibrin and are used in the diagnosis of disorders of the fibrinolytic system. One such POC D-dimer assay, (ACQT90 Flex D-dimer) reported by Sidelmann et al. uses fluorescence and D-dimer antibody binding to determine its concentration in whole blood. ${ }^{51} \mathrm{~A}$ comparison of the effectiveness of five different commercially available D-dimer assays was executed by Geersing et al. who reported high sensitivities for all assays analysed and recommended their suitability for incorporation into the GP setting for exclusion of venous thrombosis. ${ }^{52}$ These devices incorporate tests ranging from immunochromatography to enzyme-based immunoassays.

\section{Platelet function assays}

Platelets aggregate at a site of injury to form a primary platelet plug, in what can be described as a multi-step process, prior to thrombin generation and the formation of the haemostatic plug. ${ }^{7}$ It is the exposure of various subendothelial factors (collagen, laminin, fibrinogen) that promote platelet adhesion..$^{53,54}$ Some important features of platelet adhesion include the shear rate within the blood vessels and the release of the glycoprotein von Willebrand factor (VWF). Under high shear, it is VWF that promotes platelet clumping. ${ }^{54}$

Disorders that arise due to platelet abnormalities include thrombocythemia, thrombocytosis and thrombocytopenia. ${ }^{55}$ Platelet function assays are used to assess abnormalities in platelet adhesion, which is central in the response to vessel damage, in addition to monitoring the efficacy of various antiplatelet drugs such as aspirin, or glycoprotein IIb/IIIa inhibitors. The importance of these assays is increasing due to the increasing number of patients on anti-platelet therapy that present with a high risk of bleeding. ${ }^{56} \mathrm{Li}$ et al. present a nice review of the platelet response to a range of pharmacological agents in whole blood on microfluidic platforms. ${ }^{57}$

Microfluidics is emerging as a key player in current research around platelet function biology due to its controlled hemodyanmics. ${ }^{57,58}$ Schoeman et al. report a PDMS-based microfluidic model of haemostasis, whereby thrombus formation is mimicked within the channel. The impact of the antiplatelet P2Y12 receptor inhibitor on platelet activation was also determined, indicating the potential of such a device in the analysis of antiplatelet effects. ${ }^{59}$ The platelet response to Abciximab, another anti-platelet drug, was analysed in a well-plate microfluidic device that also accounted for shear flow, yielding a more realistic insight into platelet aggregation. ${ }^{60}$

The clinical impact of microfluidic devices has also been reported in the literature with many devices reporting on their use with samples from haemophiliac patients, reflecting real world applications. Measuring platelet deposition and fibrin formation, the collagen coated devices range from hard plastic ${ }^{61}$ to soft lithography generated PDMS chips, ${ }^{58,62}$ emphasising low cost and easy fabrication methodologies.

Innovative designs that improve upon previous iterations of devices are also prevalent in the literature. One such device incorporates a herringbone mixer on-chip to allow for recalcification of citrated whole blood, eliminating the preprocessing step. The mixer does not cause platelet activation, thus thrombus formation can be monitored for up to 30 minutes, to gain extra patient data, compared to standard flow assays that can only be visualised for $3-10$ minutes (Fig. 3). ${ }^{63}$

\section{Conclusions}

The rate at which microfluidics research is advancing is impressive. Simple, low-cost tools and materials (plastics and paper) are the current trends in this space. And combined with low cost readers (smartphones, LEDs, CCD cameras) they become an attractive package for POCT. Makerspacers have recently been described as facilitators in the application of 
microfluidics to the life sciences, opening up opportunities that will ameliorate the success of the transition of POC devices to market. ${ }^{5}$ These physical spaces provide improved access to lowcost fabrication technologies that encourage microdevice development for biological applications.

The optimisation and subsequent validation of POC technology does present challenges in terms of performance characteristics such as sensitivity, specificity and reproducibility and operational characteristics including initial cost and assay stability. As with any new medical device issues around clinical acceptance and adoption into practice will depend on economic factors such as patient demand. ${ }^{9}$ Technologies in POCT are developing rapidly, with state-of-the art and ingenious concept designs. The injection of such technologies onto the commercial market does however present with challenges. Barriers include the slow transition of these microdevices within the small research laboratory to large scale mass production, the quality of mass produced devices, and the associated cost of upscaling these technologies. ${ }^{2}$ Government regulation and issues around intellectual property also feature as potential barriers to commercialisation. ${ }^{9}$

Innovative materials such as nanoparticles, nanostructures and nanowires for device development, nanoparticle and labelfree detection of biomolecules are just a snapshot of what we can expect to see in future miniaturised devices. ${ }^{64}$ What emerges from the literature around coagulation microfluidics is the absence of (1) a robust global assay of haemostasis and (2) a coagulation device that offers multiplexing. POC multiplex haematology would offer clinicians efficiency and convenience, while improving patient outcomes at the point-of-care ${ }^{65}$ Incremental improvements in assay and technology development, as outlined in this review, can only prove beneficial to the haemostasis and coagulation community.

\section{Conflicts of interest}

There are no conflicts of interest to declare.

\section{References}

1 V. Gubala, L. F. Harris, A. J. Ricco, M. X. Tan and D. E. Williams, Anal. Chem., 2012, 84, 487-515.

2 A. St John and C. P. Price, Clin. Biochem. Rev., 2014, 35, 155167.

3 S. L. Diamond, Cell. Mol. Bioeng., 2017, 10, 1-2.

4 G. M. Whitesides, Nature, 2006, 442, 368-373.

5 D. I. Walsh, D. S. Kong, S. K. Murthy and P. A. Carr, Trends Biotechnol., 2017, 35, 383-392.

6 L. F. Harris, P. Rainey, T. L. Lindahl and A. J. Killard, Anal. Methods, 2016, 8, 6500-6505.

7 S. A. Smith, R. J. Travers and J. H. Morrissey, Crit. Rev. Biochem. Mol. Biol., 2016, 50, 326-336.

8 G. Lippi, E. J. Favalaro, M. Franchini and G. C. Guidi, Semin. Thromb. Hemostasis, 2009, 35, 9-22.

9 L. F. Harris, V. Castro-López and A. J. Killard, Trends Anal. Chem., 2013, 50, 85-95.

10 M. D. Lancé, Thromb. J., 2015, 13, 1-6.
11 J. Zilberman-Rudenko, J. Sylman, H. Lakshmanan, O. McCarty and J. Maddala, Cell. Mol. Bioeng., 2017, 10, 16-29.

12 A. Jain, A. Graveline, A. Waterhouse, A. Vernet, R. Flaumenhaft and D. E. Ingber, Nat. Commun., 2016, 7, $1-10$.

13 S. Zhu and S. L. Diamond, Thromb. Res., 2014, 134, 13351343.

14 B. R. Branchford, C. J. Ng, K. B. Neeves and J. Di Paola, Thromb. Res., 2015, 136, 13-19.

15 T. V. Colace, P. F. Fogarty, K. A. Panckeri, R. Li and S. L. Diamond, J. Thromb. Haemostasis, 2014, 12, 147-158.

16 A. St. John, in Point of Care Testing, ed. C. P. Price, A. St. John and J. M. Hicks, American Association for Clinical Chemistry Inc., Washington, USA, 2nd edn, 2004, pp. 31-45.

17 M. J. Pugia and C. P. Price, in Point of Care Testing, ed. C. P. Price, A. St. John and J. M. Hicks, American Association for Clinical Chemistry Inc., Washington, USA, 2nd edn, 2004, pp. 13-30.

18 L. F. Harris, P. Rainey, V. Castro-López, J. S. O'Donnell and A. J. Killard, Analyst, 2013, 138, 4769-4776.

19 B. Kim, M. L. Quan, R. Y. Goh, J. E. Kim, K. S. Woo, M. H. Kim and J. Y. Han, Lab. Med., 2013, 44, 319-323.

20 M. Levi and B. J. Hunt, J. Thromb. Haemostasis, 2015, 13, 1960-1967.

21 J. Arvind, Coagulation or lysis assays by impedance, Pat. No. US6673622B1, 1999, 1-16.

22 Siemens-Healthcare, Xprecia Stride ${ }^{\circledR}$ Coagulation Analyzer, https:/www.healthcare.siemens.com/coagulation/ coagulation/xprecia-stride-analyzer, 2018.

23 M. Vacas, P. J. Lafuente, I. Unanue and J. A. Iriarte, Hematol. J., 2004, 5, 35-38.

24 J. Hirsh, M. O'Donnell and J. I. Weitz, Blood, 2005, 105, 453463.

25 L. F. Harris, V. Castro-López, N. Hammadi, J. S. O'Donnell and A. J. Killard, Talanta, 2010, 81, 1725-1730.

26 J. Hirsh, T. E. Warkentin, S. G. Shaughnessy, S. S. Anand, J. L. Halperin, R. Raschke, C. Granger, E. M. Ohman and J. E. Dalen, Chest, 2001, 119, 64S-94S.

27 S. Middeldorp, Thromb. Res., 2008, 122, 753-762.

28 R. S. Kazmi and B. A. Lwaleed, Br. J. Clin. Pharmacol., 2011, 72, 593-603.

29 M. van Montfoort and J. Meijers, Thromb. Haemostasis, 2013, 110, 223-232.

30 V. Govindarajan, S. Zhu, R. Li, Y. Lu, S. L. Diamond, J. Reifman and A. Y. Mitrophanov, Biophys. J., 2018, 114, 978-991.

31 R. Gorkin, J. Park, J. Siegrist, M. Amasia, B. S. Lee, J.-M. Park, J. Kim, H. Kim, M. Madou and Y.-K. Cho, Lab Chip, 2010, 10, 1758-1773.

32 S. Haeberle, T. Brenner, R. Zengerle and J. Ducree, Lab Chip, 2006, 6, 776-781.

33 X. B. Zhang, Z. Q. Wu, K. Wang, J. Zhu, J. J. Xu, X. H. Xia and H. Y. Chen, Anal. Chem., 2012, 84, 3780-3786.

34 H. Li, D. Han, G. M. Pauletti and A. J. Steckl, Lab Chip, 2014, 14, 4035-4041. 
35 M. T. Ganter and C. K. Hofer, Anesth. Analg., 2008, 106, 13661375.

36 L. Bochsen, B. Wiinberg, M. Kjelgaard-Hansen, D. A. Steinbrüchel and P. I. Johansson, Thromb. J., 2007, 5, 3.

37 D. Bolliger and K. Tanaka, Semin. Thromb. Hemostasis, 2017, 43, 386-396.

38 D. Maji, M. A. Suster, E. Kucukal, U. D. S. Sekhon, A. Sen Gupta, U. A. Gurkan, E. X. Stavrou and P. Mohseni, IEEE Transaction on Biomedical Circuits and Systems, 2017, 11, 1459-1469.

39 T. P. Vikinge, K. M. Hansson, P. Sandström, B. Liedberg, T. L. Lindahl, I. Lundström, P. Tengvall and F. Höök, Biosens. Bioelectron., 2000, 15, 605-613.

40 L. Harris, R. S. Lakshmanan, V. Efremov and A. J. Killard, in Medical Biosensors for Point of Care (POC) Applications, ed. R. J. Narayan, Elsevier Woodhead Publishing, Duxford, UK, 1st edn, 2017, pp. 203-227.

41 L. Müller, S. Sinn, H. Drechsel, C. Ziegler, H. P. Wendel, H. Northoff and F. K. Gehring, Anal. Chem., 2010, 82, 658663.

42 M. Hussain, H. Northoff and F. K. Gehring, Biosens. Bioelectron., 2015, 66, 579-584.

43 M. H. Stefan Sinn, J. Biosens. Bioelectron., 2013, 4, 4-9.

44 R. S. Lakshmanan, V. Efremov, J. S. O. Donnell and A. J. Killard, Anal. Bioanal. Chem., 2016, 408, 6581-6588.

45 S. Sinn, L. Müller, H. Drechsel, M. Wandel, H. Northoff, G. Ziemer, H. P. Wendel and F. K. Gehring, Analyst, 2010, 135, 2930-2938.

46 M. Hussain, H. P. Wendel, K. Schmidt, E. Langer, M. K. Körber, O. Faul, H. Northoff, C. von Heymann and F. K. Gehring, Biosens. Bioelectron., 2018, 104, 15-20.

47 A. de la Escosura-Muñiz, W. Chunglok, W. Surareungchai and A. Merkoçi, Biosens. Bioelectron., 2013, 40, 24-31.

48 H. Xu, K. Gorgy, C. Gondran, A. Le Goff, N. Spinelli, C. Lopez, E. Defrancq and S. Cosnier, Biosens. Bioelectron., 2013, 41, 90-95.

49 C. Sun, Q. Han, D. Wang, W. Xu, W. Wang, W. Zhao and M. Zhou, Anal. Chim. Acta, 2014, 850, 33-40.
50 K. Evans, Z. Amenhotep, D. Dawson, H. Waters and J. Ardern, in The Immunoassay Handbook, ed. D. Wild, Elsevier Ltd., Oxford, UK, 3rd edn, 2013, pp. 795-816.

51 J. J. Sidelmann, J. Gram, A. Larsen, K. Overgaard and J. Jespersen, Thromb. Res., 2010, 126, 524-530.

52 G. J. Geersing, D. B. Toll, K. J. M. Janssen, R. Oudega, M. J. C. Blikman, R. Wijland, K. M. K. de Vooght, A. W. Hoes and K. G. M. Moons, Clin. Chem., 2010, 56, 1758-1766.

53 L. K. Jennings and J. Kotha, Drug Dev. Res., 2013, 74, 587593.

54 M. Nagy, J. W. M. Heemskerk and F. Swieringa, Platelets, 2017, 28, 441-448.

55 M. Krishnegowda and V. Rajashekaraiah, Blood Coagulation Fibrinolysis, 2015, 26, 479-491.

56 R. Paniccia, R. Priora, A. A. Liotta and R. Abbate, Vasc. Health Risk Manage., 2015, 11, 133-148.

57 R. Li, T. Grosser and S. L. Diamond, Platelets, 2017, 28, 457462.

58 T. Colace, P. F. Fogarty, K. A. Panckeri, R. Li and S. L. Diamond, J. Thromb. Haemostasis, 2014, 12, 147-158.

59 R. Schoeman, K. Rana, N. Danes, M. Lehmann, J. Di Paola, A. Fogelson, K. Leiderman and K. Neeves, Cell. Mol. Bioeng., 2017, 10, 3-15.

60 C. G. Conant, J. T. Nevill, Z. Zhou, J. F. Dong, M. A. Schwartz and C. Ionescu-Zanetti, J. Lab. Autom., 2011, 16, 148-152.

61 F. Swieringa, M. J. E. Kuijpers, M. M. E. Lamers, P. E. J. van der Meijden and J. W. M. Heemskerk, Haematologica, 2015, 100, 748-756.

62 A. A. Onasoga-Jarvis, K. Leiderman, A. L. Fogelson, M. Wang, M. J. Manco-Johnson, J. A. Di Paola and K. B. Neeves, PLoS One, 2013, 8, 1-12.

63 M. Lehmann, A. M. Wallbank, K. A. Dennis, A. R. Wufsus, K. M. Davis, K. Rana and K. B. Neeves, Biomicrofluidics, 2015, 9, 9-13.

64 D. Giljohann and C. A. Mirkin, Nature, 2009, 461-464.

65 C. M. Curtis, G. J. Kost, R. F. Louie, R. J. Sonu, E. B. Ammirati and S. Sumner, Point Care, 2012, 11, 140-145. 